\title{
A tabu search approach for mixed-model parallel assembly line balancing problem (type II)
}

\begin{abstract}
There are two types of assembly line balancing problems (ALBPs), which have received a great attention in the literature during the past four decades: types I and II. Both versions of ALBP are NP-hard and cannot be solved by exact methods. This paper presents an efficient tabu search (TS) metaheuristic approach for allocating tasks to balance the mixed-model production through the parallel assembly lines. Firstly, an initial solution is determined by a heuristic algorithm. Secondly, an optimum cycle time for each line in the mixed-model parallel assembly line (MMPAL) is calculated through the TS. The developed methodology, tabu search mixed-model parallel assembly line balancing (TMMPALB) model, is tested on test problems producing three and four products. Computational experiments show that the TMMPALB produces good enough solutions in case of MMPALs. The results of the proposed procedure improve cycle times of 77 test problems out of 87 .
\end{abstract}

Keyword: Assembly line balancing; Industrial engineering; Mixed model product; PALs; Parallel assembly lines; Tabu search 